\title{
A Model of Self-Avoiding Random Walks for Searching Complex
}

\section{Networks}

\author{
Víctor M. López Millán ${ }^{1}$, Vicent Cholvi ${ }^{2}$, Luis López ${ }^{3}$, and Antonio Fernández Anta ${ }^{4}$ \\ ${ }^{1}$ Universidad CEU San Pablo, Madrid, Spain (Assistant Professor) \\ ${ }^{2}$ Universitat Jaume I, Castellón, Spain (Associate Professor) \\ ${ }^{3}$ Universidad Rey Juan Carlos, Madrid, Spain (Assistant Professor) \\ ${ }^{4}$ Institute IMDEA Networks, Madrid, Spain (Full Professor)*
}

May 28, 2011

\begin{abstract}
Random walks have been proven useful in several applications in networks. Some variants of the basic random walk have been devised pursuing a suitable trade-off between better performance and limited cost. A self-avoiding random walk (SAW) is one that tries not to revisit nodes, therefore covering the network faster than a random walk. Suggested as a network search mechanism, the performance of the SAW has been analyzed using essentially empirical studies. A strict analytical approach is hard since, unlike the random walk, the SAW is not a Markovian stochastic process. We propose an analytical model to estimate the average search length of a SAW when used to locate a resource in a network. The model considers single or multiple instances of the resource sought and the possible availability of one-hop replication in the network (nodes know about resources held by their neighbors). The model characterize networks by their size and degree distribution, without assuming a particular topology. It is, therefore, a mean-field model, whose applicability to real networks is validated by simulation. Experiments with sets of randomly built regular networks, Erdős-Rényi networks, and scale-free networks of several sizes and degree averages, with and without one-hop replication, show that model predictions
\end{abstract}

*Partially funded by Comunidad de Madrid under grant S2009TIC-1692 and Spanish MICINN under grant TIN2008-06735-C02-01. 
are very close to simulation results, and allow us to draw conclusions about the applicability of SAWs to network search.

Keywords: self-avoiding random walk, random walk, network search, resource location, one-hop replication, average search length

\section{Introduction}

A random walk in a network is a simple routing mechanism that chooses the next node in the route uniformly at random among the neighbors of the current node. Although naive, this mechanism proves useful especially in situations where there is no complete knowledge about the network or where the network changes frequently, like the Internet, the WWW, peer-to-peer $(\mathrm{P} 2 \mathrm{P})$ networks and wireless ad-hoc networks. Some of the advantages derived from its simplicity are that it needs little processing power in the nodes and that it requires only local information, avoiding the bandwidth overhead produced by the exchange of routing information among nodes. Applications of random walks include routing, searching, network sampling, network construction and network characterization $[2,3,8,11,14,16,17,22,23,25,26,27,35,36,40]$.

Random walks on graphs have been extensively analyzed in Mathematics [19, 24, 31], where they are typically modelled as Markov chains, leading to many interesting results that include bounds on the cover time $[4,7,15,20,30,42]$ (i.e., the number of steps to visit all, or a fraction of, the nodes in the graph), and bounds on the access time $[9,33]$ (the expected number of steps to reach node $j$ starting from node $i$ ). These results are frequently based on the spectral properties of the adjacency matrix of the graph and of the transition matrix of the random walk. Random walks have also received much attention from the Physics community, since they reflect the dynamics of many natural systems including protein interactions, polymer chains, communication networks, and social networks.

The basic behavior of a random walk can be modified in a number of ways to optimize its performance metrics on networks. Costa and Travieso [13] study the network coverage of three types of random walks: traditional, preferential to untracked links, and preferential to unvisited nodes. They find that the latter is the best strategy in covering the network nodes, in both random (Erdős-Rényi) networks and scale-free networks. Yang [41] studies the search performance of five 
random walk variations: no-back (NB), no-triangle-loop (NTL), no-quadrangle-loop (NQL), selfavoiding (SA) and high-degree-preferential self-avoiding (PSA). He finds that all algorithms achieve similar performance in random networks, while the self-avoiding walk outperforms the others in scale-free networks and small-world networks. Both results suggest that a random walk that tries to not revisit nodes is an interesting alternative to pure random walks for network search. Our work focuses on using this type of walk for searching for resources in a network. More concretely, we define a walk that chooses the next node to be visited uniformly at random among the unvisited neighbors of the current node. If no unvisited neighbor exists, the next node is chosen uniformly at random among all the neighbors of the current node. The walk proceeds until it finds the resource searched for. We will refer to such a walk as a self-avoiding walk (SAW) in this paper.

Our model of SAW is in fact akin to the true SAW defined in [5] at short times, becoming a simple random walk at long times. The true (or myopic) SAW is defined as the stochastic process which chooses the next node to be visited among the neighbors of the current node with probability proportional to a negative exponential of the number of times visited. Our definition of SAW differs from this one in that the next node is chosen uniformly at random among the unvisited neighbors or, if none, among all neighbors, regardless of how often they have been visited in the past. This means that our SAW model loses its self-avoiding properties at long times, while the true SAW keeps them at all times.

The analysis of the self-avoiding walk with analytical tools is hard since, unlike the pure random walk, it cannot be modelled as a Markovian stochastic process. Therefore, many questions on SAWs have not yet been answered in an analytical manner. In addition, using analytical results in some real scenarios is impractical when the adjacency matrix of the network is too large or simply unknown. In Mathematics, a SAW is defined as a random walk restricted not to intersect with itself. Note that this definition is more restrictive than the one established above in networks, since such a walk never revisits a node, having therefore finite length always. We will refer to this type of walk as strict $S A W$, to avoid confusion with the SAW we are interested in to search complex networks. Available results on strict SAWs are compiled in [38]. This work also includes results on a less restrictive version called the weakly self-avoiding walk, in which intersections are not disallowed but discouraged. In this type of SAWs, the more intersections in a walk, the less probable it is to occur. Our definition of SAW differs from the weakly SAW in that it always tries to avoid already 
visited nodes.

Works from the mathematical point of view like the ones referenced and others $[21,28,39,38]$, study SAWs in $d$-dimensional lattices $\left(Z^{d}\right)$. Results include the behavior of the number of SAWs with $n$-steps in the lattice and of the mean-square displacement (the average distance between the end and the origin of a walk). In complex networks, questions have been addressed mostly through empirical approaches. The previously cited works by Costa and Travieso [13], and Yang [41] use numerical simulations. Mean access times in lattices with embedded scale-free networks providing long-range shortcuts have been obtained in [10]. In [18], strict SAWs are studied in scale-free networks, obtaining approximate analytical expressions for the mean number of SAWs starting from a generic node, and for the average maximum length of such walks over statistically independent networks. Simulation results support their approximate analytical calculations.

\subsection{Contributions}

This paper studies SAW as a search mechanism in communication networks. Our main contribution is an analytical model that estimates the average search performance of SAWs in complex networks with or without one-hop replication, and where a number of instances of the resource sought are present. In a one-hop replication network, a node knows about the resources held by its neighbors. Therefore, to find a resource in such a network it suffices to visit a node that holds it or any of its neighbors, whereas the walk must visit a node that holds the resource if one-hop replication is not available. One-hop replication is an interesting feature in a communication network, since it contributes to reducing search lengths at limited cost. It has been included as part of search mechanisms in P2P networks [11, 12, 29, 35].

In particular, our model estimates the network coverage attained at each hop of the walk and uses this to estimate the average search length. Although our definition of a SAW coincides with those of a walk preferential to unvisited nodes in [13] and of a self-avoiding (SA) walk in [41], our work differs from them in that we propose an analytical model to predict network coverage and search performance, whereas their evaluations are only based on simulation results. In addition, [13] evaluates network coverage (not network search).

We follow the approach that Rodero-Merino et al. [35] apply to "pure" random walks in onehop replication networks, i.e., we derive recurrence formulas that estimate model magnitudes as 
functions of their values at the previous hop. The model is based on networks determined only by their size and degree distribution, with no particular topology assumed. Therefore, it is, as the one in [35], a mean-field model.

The accuracy of the model predictions has been assessed using simulations. Three types of random networks have been considered for our experiments: (1) regular networks, where the degree is constant for all nodes, (2) Erdős-Rényi (ER) networks, also called random networks, where there is a fixed probability that a node is linked to any other node in the network, and (3) scale-free networks, where the degree distribution obeys a power-law behavior in the node degree. Scale-free networks are of special interest since many real communication networks exhibit power-law degree distributions, including the Internet, the WWW, and P2P networks $[1,6,34,37]$.

The networks in the experiments are built using the random mechanism proposed by Newman et al. [32] for networks with arbitrary degree distributions. For each network type, results are averaged over all searches performed in a network, and then over all the networks built. We have found that the model predictions are good approximations of the values obtained this way. Therefore, the model proposed is useful to predict average SAW search performance in large networks built randomly, as is the case, for example, of unstructured P2P resource-sharing systems.

Finally, we contribute some useful conclusions from the comparison of the performance of a SAW and a pure random walk for searching networks. As an example, it has been found, in accordance with $[13,41]$, that the SAW outperforms the pure random walk by obtaining shorter searches on the average, especially in scale-free networks. However, we have noticed that the performance gain is large in networks without one-hop replication, but it is sensibly smaller for networks with onehop replication. This is a consequence of the observed fact that, in one-hop replication networks, an average random walk covers nodes (i.e., visited nodes and neighbors of them) almost as fast as an average SAW. This means that the effect on average search length of one-hop replication is nearly equivalent to that of trying not to revisit nodes. This observation suggests that SAWs are an interesting alternative to pure random walks for searching networks without one-hop replication, which avoids the overhead of updating information about the neighbors' resources.

Summarizing, our work provides the following contributions:

- A mean-field model for predicting average network coverage and search lengths achieved by 
SAW in randomly built networks characterized by their size and degree distribution. The model takes into account the existence of one-hop replication and the multiplicity of instances of the resource searched for.

- An extension of the previous RW model proposed in [35] to support networks without one-hop replication and with more than one instance of the resource searched for.

- A study of the search performance of SAW in networks with one-hop replication. Simulations are used to validate the model predictions. Results have been compared with those of random walks in the same type of network and with search performance of RW and SAW in networks without one-hop replication.

- The observation that SAW achieves large reductions in the average search length with respect to RW in networks without one-hop replication, while reductions are moderate in networks with one-hop replication. These reductions decrease as the average degree of the network grows, except for scale-free networks without one-hop replication, where the reductions increase with the average degree.

The rest of the paper is organized as follows. Section 2 presents our analytical model for the estimation of network coverage and search lengths of self-avoiding walks. Section 3 analyzes the performance of the SAW in comparison with that of a pure random walk in networks with and without one-hop replication. Simulation results are provided to validate the model derived in the previous section. Finally, Section 4 states our conclusions and outlines some future work lines.

\section{The Analytical Model}

\subsection{Definitions and Assumptions}

Let $G(V, E)$ be an undirected graph representing a communication network where $V$ is the set of its nodes and $E$ is the set of its links. The network is assumed to be connected (i.e., there is some path connecting every pair of different nodes in the network). The network is characterized solely by the number of nodes $(N)$ and by its degree distribution $p_{k}$, where $p_{k}$ is the probability that a node chosen uniformly at random has degree $k$. If $n_{k}$ denotes the number of $k$-degree nodes in the 
network (i.e., $\sum_{k} n_{k}=N$ ), then $p_{k}=n_{k} / N$. An attachment point is a point in a node where one of the two ends of a link is attached. Therefore, each node has a number of attachment points equal to its degree, and the total number of attachment points in the network is $S=\sum_{k} k n_{k}$, which is also twice the total number of links in the network. Auto-links (links connecting a node to itself) and multilinks (more than one link between two nodes) are disallowed [35, 18].

The proposed model does not take into account the topology of $G$ to characterize networks. This means that it is in fact a mean-field model, whose accuracy is checked using simulation experiments. Empirical results for sets of regular, ER and scale-free networks built randomly show that model predictions are good approximations for those classes of networks. However, we believe it is possible to find classes of networks for which the structure is more important than the degree distribution, and for which the mean-field model predictions are not good approximations of real values.

The fact that the model does not consider network topology is reflected in the formalism in that it is assumed that given any attachment point of an arbitrary node $i$, the link departing from it can take us to any other attachment point (of a node $j \neq i$ ) in the network with equal probability. This condition, used also in the mean-field study in [35], is true for networks built with the random mechanism proposed by Newman [32].

We define a self-avoiding walk (SAW) as a random walk governed by the rules: $\left(R_{1}\right)$ if all neighbors of the current node have been already visited, choose the next node uniformly at random among all neighbors (including the one the walk came from), and $\left(R_{2}\right)$ if there is at least one unvisited neighbor of the current node, choose the next node uniformly at random among the unvisited neighbors.

We assume that the walk will search the network for a particular resource, starting at a node chosen uniformly at random from all network nodes (at hop $h=0$ ). There are $R>0$ instances of the resource sought. This allows us to model scenarios with multiplicity of resource instances such as peer-to-peer (P2P) networks, where several peers contain the same file [17]. We assume that these instances are held by (different) nodes chosen uniformly at random. In networks without one-hop replication, the search will finish when the walk visits any node holding an instance of the resource. In networks with one-hop replication, visiting a neighbor of any node holding the resource will finish the search.

Our ultimate goal is to obtain an expression for the average search length (denoted $\bar{h}$ ), defined 
as the average number of hops it takes to find an instance of the resource sought. This magnitude depends on the number of different nodes that the walk has visited (denoted $V(h)$ ) or covered (denoted $C(h)$ ) up to and including hop $h$. In turn, $V(h)$ and $C(h)$ depend on a number of auxiliary magnitudes that capture the behavior of the SAW. These magnitudes are defined in Table 1 and their expressions are derived in the next paragraphs.

\section{$2.2 \quad$ Visited nodes}

We start by estimating $P_{w}^{k}(h)$, the probability of visiting any node $j$ of degree $k$ at hop $h$, given that the walk is currently at some node $i$. Two different cases need to be considered: (1) all neighbors of $i$ have already been visited, and (2) at least one neighbor of $i$ has not been visited yet, corresponding to rules $R_{1}$ and $R_{2}$ of the SAW, respectively.

- Case 1: This probability is the ratio of "positive" attachment points and possible attachment points. We consider attachment points belonging to nodes of degree $k$ to be positive and all attachment points in the network to be possible ${ }^{1}$. The probability of visiting any node $j$ of degree $k$ at hop $h$, conditioned on the fact that all neighbors of $i$ have already been visited is then:

$$
\begin{aligned}
P_{w, \text { anv }}^{k}=\frac{k n_{k}}{S} & =\frac{k n_{k}}{\sum_{j} j n_{j}}=\frac{k p_{k}}{\sum_{j} j p_{j}}=\frac{k p_{k}}{\bar{k}}, \text { for } h>0, \\
P_{w, \text { anv }}^{k}(0) & =p_{k} .
\end{aligned}
$$

Here, we are using our assumption that a given attachment point of $i$ can take us to any attachment point in the network with equal probability. We are also taking into account the fact that the attachment point of $i$ will be chosen uniformly at random for hop $h$, as $R_{1}$ states. Note that this probability is not a function of the hop $h$ (except for $h=0$ ), since the behavior is that of a random walk, whose next state depends only on the current state and not on the

\footnotetext{
${ }^{1}$ To be fully precise, the attachment points of $i$ should not be considered either as possible or positive (in case the degree of $i$ is $k$ ), since auto-links are not allowed. However, that would introduce dependency on the degree of $i$, cluttering the analysis. Results for large randomly generated regular, ER and scale-free networks show that no significant error is introduced by this simplification.
} 


\begin{tabular}{|c|c|}
\hline Magnitude & Definition \\
\hline$P_{w}^{k}(h)$ & $\begin{array}{l}\text { Probability of the walk arriv- } \\
\text { ing at any node of degree } k \text { at } \\
\text { hop } h \text {, with } P_{w}^{k}(0)=p_{k} \text {. }\end{array}$ \\
\hline$P_{w, a n v}^{k}$ & $\begin{array}{l}\text { Probability of the walk arriv- } \\
\text { ing at any node of degree } k \text { at } \\
\text { any hop, conditioned on that } \\
\text { all the neighbors of the pre- } \\
\text { vious node have already been } \\
\text { visited ( "anv" = all neighbors } \\
\text { visited). }\end{array}$ \\
\hline$P_{w, \text { nanv }}^{k}(h)$ & $\begin{array}{l}\text { Probability of the walk arriv- } \\
\text { ing at any node of degree } k \\
\text { at hop } h \text {, conditioned on that } \\
\text { at least one neighbor of the } \\
\text { previous node has not been } \\
\text { visited yet ("nanv" = not all } \\
\text { neighbors visited). }\end{array}$ \\
\hline$P_{a n v}^{k}(h)$ & $\begin{array}{l}\text { Probability that all the neigh- } \\
\text { bors of the node reached at } \\
\text { hop } h \text { have already been vis- } \\
\text { ited by the walk ( "anv" = } \\
\text { all neighbors visited), condi- } \\
\text { tioned on that the degree of } \\
\text { that node is } k \text {. }\end{array}$ \\
\hline$P_{a n v}(h)$ & $\begin{array}{l}\text { Probability that all the neigh- } \\
\text { bors of the node (of any de- } \\
\text { gree) reached at hop } h \text { have } \\
\text { already been visited by the } \\
\text { walk ("anv" = all neighbors } \\
\text { visited). }\end{array}$ \\
\hline$P_{n v}(h)$ & $\begin{array}{l}\text { Probability that a neighbor } \\
\text { (any) of the node reached at } \\
\text { hop } h \text { (excluding the previous } \\
\text { node) has already been visited } \\
\text { by the walk ( } n v "=\text { neighbor } \\
\text { visited). }\end{array}$ \\
\hline
\end{tabular}

Table 1: Definitions of the auxiliary magnitudes of the model. 
past history of the walk. For $h=0$, the node is chosen uniformly at random among all the nodes in the network, so the probability of reaching a node of degree $k$ is $p_{k}$.

- Case 2: Positive cases are now only those attachment points belonging to non-visited nodes of degree $k$. Similarly, possible attachment points are now only those belonging to all non-visited nodes. Let us denote by $V^{k}(h)$ the average number of different nodes of each degree $k$ visited by the walk up to and including hop $h$. We obtain the number of non-visited nodes from the number of visited nodes at the previous hop $\left(V^{k}(h-1)\right)$. Thus, the probability of visiting any node $j$ of degree $k$ at hop $h$, conditioned on the fact that not all neighbors of $i$ have already been visited is:

$$
\begin{aligned}
P_{w, \text { nanv }}^{k}(h)=\frac{k\left(n_{k}-V^{k}(h-1)\right)}{S-\sum_{j} j V^{j}(h-1)} & =\frac{k\left(p_{k}-\frac{V^{k}(h-1)}{N}\right)}{\bar{k}-\sum_{j} j \frac{V^{j}(h-1)}{N}}, \text { for } h>0, \\
P_{w, \text { nanv }}^{k}(0) & =p_{k} .
\end{aligned}
$$

This probability does depend on the hop $h$ since the number of visited nodes increases as the walk proceeds through the network.

Using Equations (1) and (2), and taking into account that $P_{a n v}(h-1)$ and $\left(1-P_{\text {anv }}(h-1)\right)$ are the probabilities that the SAW follows $R_{1}$ and $R_{2}$ at hop $h$, respectively, we have that $P_{w}^{k}(h)$ can now be obtained as:

$$
\begin{aligned}
P_{w}^{k}(h)=P_{w, a n v}^{k} \cdot P_{a n v}(h-1) & +P_{w, n a n v}^{k}(h) \cdot\left(1-P_{a n v}(h-1)\right), \text { for } h>0, \\
P_{w}^{k}(0) & =p_{k} .
\end{aligned}
$$

On the other hand, $P_{a n v}(h)$ depends on the number of neighbors of the node reached at hop $h$ (i.e., its degree), and can be obtained from the probabilities that all neighbors of the node have already been visited conditioned on the fact that the degree of that node is $k$ :

$$
P_{a n v}(h)=\sum_{k} P_{a n v}^{k}(h) \cdot P_{w}^{k}(h)
$$


At this point, we note that:

$$
P_{a n v}^{k}(h)=\left(P_{n v}(h)\right)^{k-1} .
$$

The node from which the walk came from is excluded from the calculation (thus the exponent $k-1$ ), since it is certain that this node has already been visited. We are implicitly assuming here that each of the neighbors of the node are visited or not independently from the others. This approximation has been shown to be accurate in the types of network we consider [35].

The probability of a neighbor having been visited depends in turn on the degree of that neighbor, since nodes with higher degree have higher chance of being visited. This probability is thus calculated as:

$$
\begin{aligned}
& P_{n v}(h)=\sum_{k} P_{w, a n v}^{k} \frac{V^{k}(h-2)}{n_{k}}, \text { for } h>1, \\
& P_{n v}(0)=0 \\
& P_{n v}(1)=0 .
\end{aligned}
$$

$P_{w, \text { anv }}^{k}$ plays here the role of the probability that the neighbor is of degree $k$ (the probability of visiting a node of degree $k$ from the current node when it is chosen uniformly at random), while the fraction within the summation gives the probability of a neighbor of degree $k$ being already visited as the ratio of positive nodes and possible nodes. Here, $V^{k}(h-2)$ is used instead of $V^{k}(h)$ to exclude the node reached at hop $h$ (in case its degree is $k$ ) and the node the walk came from (in case its degree is $k$ ). The latter is certain to have been visited already as stated above, and thus does not participate in the calculation of $P_{a n v}^{k}(h)$.

$V^{k}(h)$ can now be estimated using the following recursive expression:

$$
\begin{aligned}
V^{k}(h) & =V^{k}(h-1)+P_{w, \text { nanv }}^{k}(h) \cdot\left(1-P_{a n v}(h-1)\right), \text { for } h>0, \\
V^{k}(0) & =p_{k}
\end{aligned}
$$

since a new node of degree $k$ is visited at hop $h$ with probability $P_{w, n a n v}^{k}(h)$ given that the routing algorithm follows $R_{2}$, which in turn happens with probability $\left(1-P_{a n v}(h-1)\right)$. The average total 
number of different nodes visited by the walk up to and including hop $h$ is then:

$$
V(h)=\sum_{k} V^{k}(h)
$$

\subsection{Covered Nodes}

This magnitude estimates the average number of nodes covered by the walk up to and including hop $h$, denoted by $C(h)$. A node is covered by the SAW when the walk visits that node or any one of its neighbors. The number of nodes covered by the walk allows the estimation of average search length in one-hop replication networks in the last step of our analysis, since to find a resource in such a network it suffices to cover (not to visit) the node that holds it.

We first estimate $C^{k}(h)$, the average number of different nodes of degree $k$ covered by the walk up to and including hop $h$, in order to obtain $C(h)$.

For networks with a given degree distribution, the average number of nodes of degree $k$ that a walk covers at each hop clearly depends on the routing algorithm used. An expression for $C(h)$ was obtained in [35] for a pure random walk in the same types of network as those considered in our work. It is expressed in terms of the average number of visited nodes of each degree $\left(V^{k}(h)\right)$ and the degree distribution of the network, where $V^{k}(h)$ contains the effect of the routing algorithm. Therefore, that expression is still valid if we change the random walk into a SAW provided that we use the expressions for $V^{k}(h)$ derived above for the SAW. Thus, the average number of covered nodes of degree $k$ can be written as:

$$
\begin{aligned}
& C^{k}(h)=C^{k}(h-1)+\left(\frac{k\left(n_{k}-C^{k}(h-1)\right)}{S-\sum_{j} j \cdot V^{j}(h-1)}\right) \times \sum_{j}\left(V^{j}(h)-V^{j}(h-1)\right)(j-1), \text { for } h>0, \\
& C^{k}(0)=V^{k}(0)+\bar{k} P_{w, \text { anv }}^{k} .
\end{aligned}
$$

Finally, the average number of covered nodes is obtained as:

$$
C(h)=\sum_{k} C^{k}(h)
$$




\subsection{Search Length}

The average search length is obtained from the probability of finishing the search at hop $h\left(P_{f i n}(h)\right)$, that is in turn obtained from the probability of being successful in finding the resource at hop $h$ $\left(P_{\text {suc }}(h)\right)$ :

$$
\bar{h}=\sum_{h=0}^{\infty} h \cdot P_{f i n}(h),
$$

where

$$
\begin{aligned}
& P_{\text {fin }}(h)=P_{\text {suc }}(h) \prod_{i=0}^{h-1}\left(1-P_{\text {suc }}(i)\right), \text { for } h>0, \\
& P_{\text {fin }}(0)=\frac{R}{N}
\end{aligned}
$$

Recall that there are $R$ instances of the resource, placed in different nodes. Now, $P_{\text {suc }}(h)$ depends on whether or not the networks considered are one-hop replication networks. For networks without replication, $P_{\text {suc }}(h)$ can be estimated as the relation between the number of new nodes visited at hop $h$ and the number of nodes that are still unvisited at that hop, taking into account the number of resource instances:

$$
\begin{aligned}
P_{\text {suc }}(h) & =R \frac{V(h)-V(h-1)}{N-V(h-1)}, \text { for } h>0, \\
P_{\text {suc }}(0) & =\frac{R}{N}
\end{aligned}
$$

For one-hop replication networks, $P_{\text {suc }}(h)$ can be similarly estimated as the relation between the number of new nodes covered at hop $h$ and the number of nodes that are still uncovered at that hop:

$$
\begin{aligned}
& P_{\text {suc }}(h)=R \frac{C(h)-C(h-1)}{N-C(h-1)}, \text { for } h>0, \\
& P_{\text {suc }}(0)=\frac{R}{N} .
\end{aligned}
$$

From Equations (11), (13) and (14) we can finally write the average search length in networks with no replication $\left(\bar{h}_{n r}\right)$ and in one-hop replication networks $\left(\bar{h}_{1 h r}\right)$, respectively, as: 


$$
\begin{aligned}
\bar{h}_{n r}= & \sum_{h=1}^{\infty} h\left[R \frac{V(h)-V(h-1)}{N-V(h-1)} \cdot\left(1-R \frac{V(0)}{N}\right) .\right. \\
& \left.\prod_{i=1}^{h-1}\left(1-R \frac{V(i)-V(i-1)}{N-V(i-1)}\right)\right],
\end{aligned}
$$

and

$$
\begin{aligned}
\bar{h}_{1 h r}= & \sum_{h=1}^{\infty} h\left[R \frac{C(h)-C(h-1)}{N-C(h-1)} \cdot\left(1-R \frac{C(0)}{N}\right) .\right. \\
& \left.\prod_{i=1}^{h-1}\left(1-R \frac{C(i)-C(i-1)}{N-C(i-1)}\right)\right] .
\end{aligned}
$$

Note that both $\bar{h}_{n r}$ and $\bar{h}_{1 h r}$ depend only on the network parameters, namely $N, p_{k}$ and $R$.

\section{Performance Evaluation}

In this section, we assess the accuracy of the SAW model comparing its predictions against simulation results. At the same time, we compare the performance of SAWs and pure random walks (RW) for searching complex networks. Experiments have been performed for three types of randomly built networks: regular networks, Erdős-Rényi networks (referred to as ER random networks or just random networks) and scale-free (power-law) networks. Network size has been set to $N=10^{4}$ nodes in the base experiment, and three average degrees have been used $(\bar{k}=10,20,30)$ to observe the effect of network connectivity.

ER random networks with degree averages $\bar{k}=10,20,30$ have been obtained with link probabilities $p=0.001,0.002$ and 0.003, respectively. Scale-free networks follow a power-law degree distribution $\left(p(k) \propto k^{-\gamma}\right)$ with $\gamma$ values of $2.7030,1.9958$ and 1.781 , adjusted to obtain feasible networks with average degrees $\bar{k}=10,20,30$, respectively. For this type of network, a minimum degree of 5 has been set to avoid disconnected networks due to the high numbers of low degree nodes. The degree distributions thus obtained are then used to feed the SAW and random walk models ${ }^{2}$, and to construct the networks for simulations using Newman's method. This method guarantees that each

\footnotetext{
${ }^{2}$ For the pure random walk, we have used the analytical model in one-hop replication networks proposed in [35], with modifications to include networks without one-hop replication and several instances of resources.
} 
attachment point is linked to any other attachment point in the network with equal probability, which is an assumption of the model as stated in Section 2.1.

To be able to fairly compare the predictions of the mean-field models with the experimental results, simulations have been run for a representative number of different networks $\left(10^{2}\right)$ with that degree distribution, and for a representative number of walks $\left(10^{3}\right)$ for each of those networks, resulting in a total of $10^{5}$ simulation runs for each experiment. Magnitudes are measured for each walk and values averaged over all walks in that network to obtain the network average. Network averages are in turn averaged over all networks to obtain the final average values to be compared with those estimated by the models. Variations of network averages compared to final average values have also been studied, finding that average values for individual networks are close to final network average values. Results for minimum, average, and maximum values, along with the typical deviation, are shown for the average search length later in this section.

We begin by showing results for two auxiliary magnitudes: the probability that all neighbors of a node have already been visited and the probability of visiting a node of degree $k$. These results will be shown for ER random networks only, as an illustration of the behavior of the SAW. Next, we show results for the average number of nodes visited $(V(h))$ and covered $(C(h))$ by the walks, which are determined by the previous probabilities. Finally, results for average search lengths $(\bar{h})$ are shown.

\subsection{Auxiliary magnitudes}

Figure 1(a) shows $P_{a n v}(h)$, the probability that all the neighbors of a node (of any degree) reached at hop $h$ have already been visited, in ER random networks with $\bar{k}=10$. It is an interesting starting point, since this probability can also be interpreted as the probability that the SAW actually succeeds in avoiding already visited nodes, or else it falls back to the RW behavior.

This figure clearly shows three different regions or phases, which correspond to different behaviors of the SAW. The probability that all neighbors of the node have already been visited is very low up to around 7500 hops, which is $75 \%$ the size of the network. Therefore, the SAW will visit a new node in every hop with high probability in this region. Then, the probability that all neighbors have already been visited grows rapidly in the central region of the graph (roughly between 7500 and 12500 hops). This is a phase of transition between the SAW behavior and the RW behavior. 


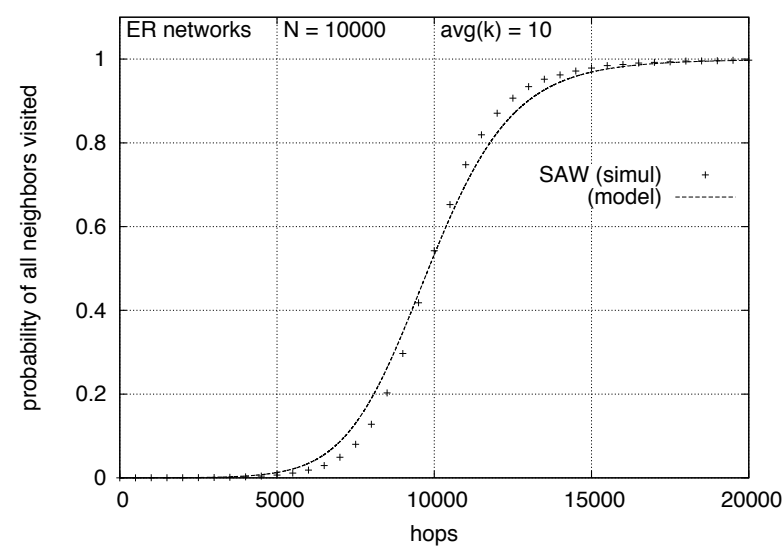

(a) Probability of all neighbors visited $\left(P_{a n v}(h)\right)$.

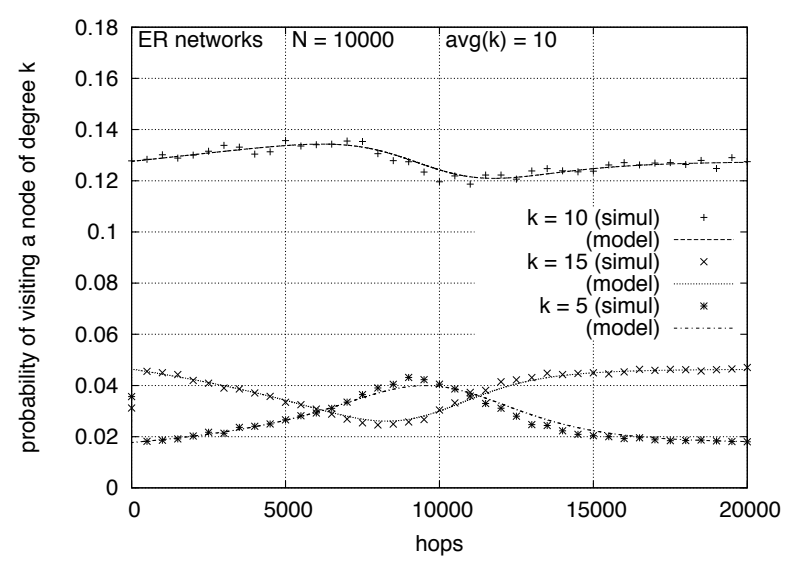

(b) Probability of visiting a node of degree $k\left(P_{w}^{k}(h)\right)$.

Figure 1: Auxiliary magnitudes of the SAW model in ER random networks with $\bar{k}=10$.

For numbers of hops greater than 12500 , the probability grows asymptotically to 1 . In this region the SAW behaves very much like a RW, since it is almost certain that all neighbors of the nodes reached have already been visited.

This figure also shows that the model predicts results with a reasonable accuracy. The more significant deviations between model and simulation results occur in the central region of the graph (transition phase). For smaller number of hops, the model is pessimistic, that is, it predicts higher probabilities that all neighbors are visited than the simulation does. This is inverted for greater number of hops. This will yield pessimistic estimations of the average number of visited and covered nodes, as will be shown in later subsections. Therefore, the model presented in Section 2 is a conservative model of the real SAW behavior.

Figure 1(b) shows $P_{w}^{k}(h)$, the probability of visiting a node of degree $k$ at hop $h$. This magnitude is interesting because it shows, like the previous one, the different phases of the algorithm. In addition, $P_{w}^{k}(h)$ is the base to estimate the average number of visited and covered nodes, which in turn are used to estimate the average search length. Curves are presented for three representative degrees: the average degree $(k=\bar{k}=10)$, a degree above the average $(k=15)$, and a degree below the average $(k=5)$. The first thing to note about this graph is that the probabilities for each degree start (low $h$ ) and finish (high $h$ ) at the same values. Indeed, when very few nodes are visited, the probability of reaching a node of a given degree is very similar to that probability of the RW model, since the next hop is chosen uniformly at random among the (non-visited) neighbors of the node. 
Likewise, when almost all nodes have been visited, the probability of visiting a node of some degree is very similar to that of the RW, since the next hop is chosen uniformly at random among the (already visited) neighbors of the node. The probability of arriving at a node of degree $k$ at any hop $\left(P_{A}^{k}\right)$ in the RW model [35] is the same as the probability of visiting a node of degree $k$ at any hop if all the neighbors of the current node have already been visited $\left(P_{w, a n v}^{k}\right)$ in the SAW model. Recall from Subsection 2.2 that this probability is not a function of $h$, since RW is a memory-less

algorithm. Therefore, in the SAW model $P_{w}^{k}(h)$ starts at $P_{w, a n v}^{k}=\frac{k p_{k}}{\bar{k}}$ for low $h$ and tends to the same value for high $h$.

In the first section of the graph (up to about 7500 hops), the SAW visits a new node at each hop with high probability. Since nodes with higher degrees are visited faster, the probability of visiting more new nodes of those degrees decrease as $h$ increases. This effect can be seen in Figure 1(b) in the curve for $k=15$. Nodes with lower degrees are visited more slowly, so the probability of visiting more new nodes of those degrees increases with $h$. This effect can also be seen in curves for $k=5$ and $k=10$.

In the second region of the graph (between 7500 and 12500 hops), the transition phase in Figure 1(a), the probability that all neighbors have been already visited grows rapidly. This will make the probability of reaching nodes of higher degrees grow to recover the level of $P_{w, a n v}^{k}$ in the third region of the graph $(h>12500)$. On the other hand, the probability of reaching nodes of lower degrees falls again to the level of $P_{w, a n v}^{k}$.

\subsection{Visited Nodes}

Figure 2(a) shows the average number of visited nodes as a function of the number of hops taken by the walk $(V(h))$ for the three types of networks with $\bar{k}=10$. As expected, curves for RW grow more slowly than curves for SAW, since the latter ones try to avoid revisiting nodes thus visiting new nodes faster than the former ones. In fact, we notice that SAW achieves a straight line with slope 1 in the first phase of the algorithm, that is, it visits a new node at each hop with a high probability up to a number of hops about $75 \%$ of the network size. From then on, new nodes are visited more slowly in the second phase until the whole network is covered in the third phase. The graph also shows that at least $99 \%$ of the network has been visited by the SAW when only (approximately) $75 \%$ has been visited by the RW. 


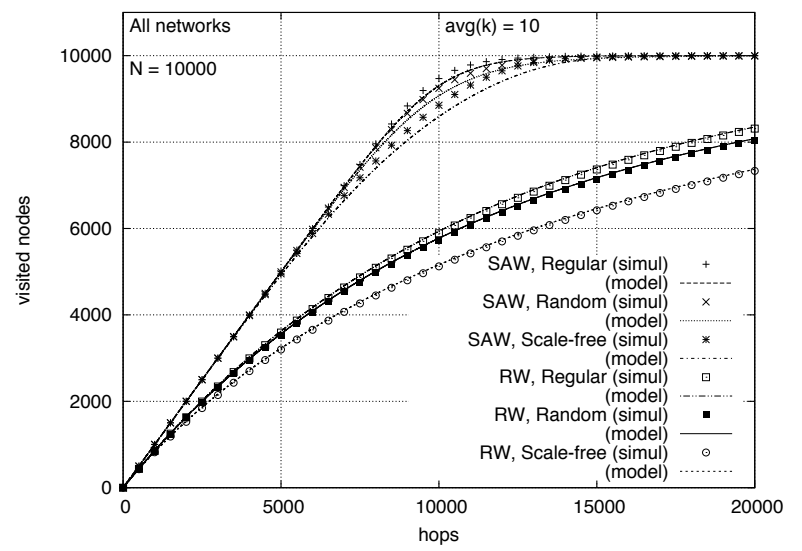

(a) All network types, $\bar{k}=10$

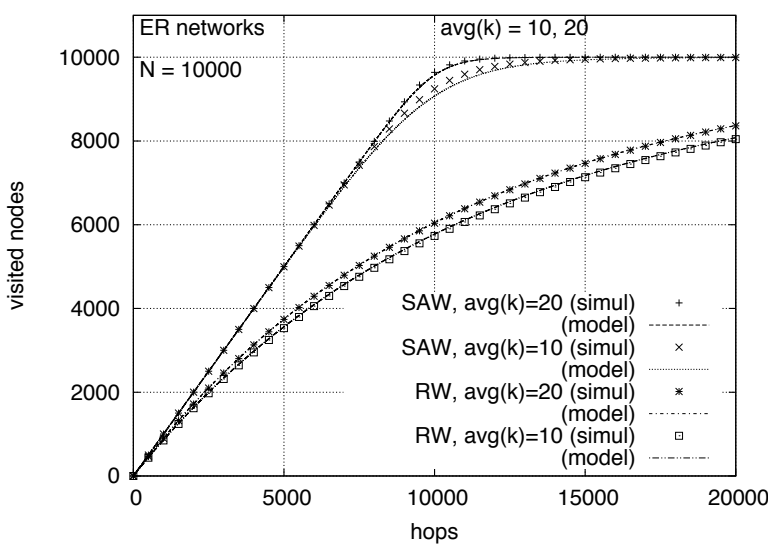

(c) ER random networks, $\bar{k}=10,20$

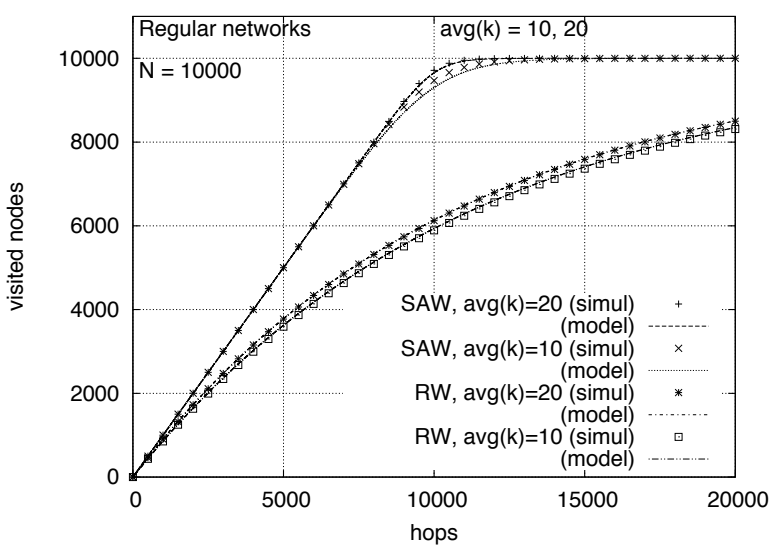

(b) Regular networks, $\bar{k}=10,20$

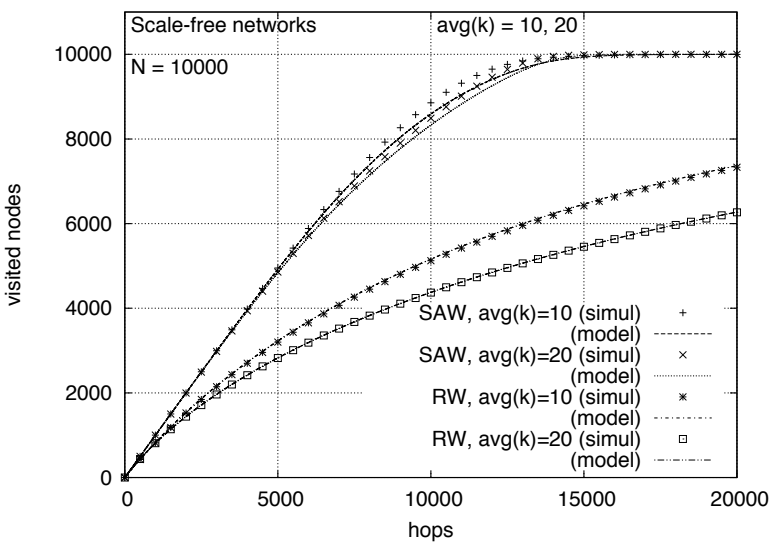

(d) Scale-free networks, $\bar{k}=10,20$

Figure 2: Average number of visited nodes of all degrees $(V(h))$. 
Both SAW and RW curves are higher for regular networks. Curves for ER random networks are slightly lower and curves for scale-free networks are still farther down. This is an effect of the different degree distributions of the three types of networks. In regular networks, all nodes are visited with the same probability, since all of them have the same degree. In random and scale-free networks a number of different degrees are present in the network. Nodes with smaller degrees are visited with smaller probability than nodes with larger degrees. It is harder for both SAW and RW to visit nodes with small degrees, resulting in a higher number of revisited nodes and thus in a slower rate of visiting new nodes. This effect is greater in scale-free networks due to the shape of power-law distributions: a large number of small degree nodes and a few nodes with a very high degree (the long tail of the distribution).

Now, we compare the average number of visited nodes in networks with average degrees $\bar{k}=10$ and $\bar{k}=20$ in Figures 2(b) to 2(d). For regular and ER random networks, curves for $\bar{k}=20$ are always higher than those for $\bar{k}=10$ for both SAW and RW. Since nodes in higher average degree networks tend to have higher degrees, it is easier to visit new nodes either randomly (RW) or trying to avoid already visited nodes (SAW), and thus the faster rate of visiting new nodes. However, the effect is reversed for scale-free networks: curves for $\bar{k}=20$ are lower than those for $\bar{k}=10$. This can be explained again by the shape of the power-law degree distribution. A higher average degree network has more very high degree nodes than a lower average degree network. Therefore, walks keep visiting these nodes with higher probability, making it more difficult to visit low degree nodes. It can also be seen that the difference is larger for RW than for SAW, since the latter tries to avoid high degree nodes once they have been visited for the first time, increasing the probability of visiting new nodes.

To further investigate this behavior in the case of SAW, we have checked the probability that all neighbors of a node have been already visited, since this is also the probability that the next node will not be a new one. In networks with higher average degree, this probability is indeed higher up to a number of hops close to the network size. This accounts for the growing divergence between curves for $\bar{k}=10$ and $\bar{k}=20$ in Figure 2(d). For subsequent hops, the probability of all neighbors being visited is higher in networks with lower average degree, explaining the convergence of the curves as they grow towards a number of visited nodes equal to the network size. The behavior of the probability of all neighbors being visited for SAW in regular and ER random networks has 
been checked to be opposite to that of scale-free networks, consistent with the observed opposite behavior of the number of visited nodes.

\subsection{Covered Nodes}

Figure 3(a) shows the average number of covered nodes as a function of the number of hops taken by the walk $(C(h))$ for networks of the three types with $\bar{k}=10$. Although SAW always achieves better rates than RW as expected, the difference here is much smaller than for the average number of visited nodes (Figure 2(a)). Although RW visits fewer new nodes than SAW, it covers almost the same number of nodes; more work needs to be done to find out why this is so. This effect reduces the difference in the performance of both algorithms when applied to searching networks with one-hop replication, as will be shown later in this section.

Figures 3(b) to 3(d) compare the average number of covered nodes in networks with average degrees $\bar{k}=10$ and $\bar{k}=20$. In regular and ER random networks, curves for $\bar{k}=20$ are higher than those for $\bar{k}=10$, the same as observed for the average number of visited nodes in Figures 2(b) and 2(c). In scale-free networks, the curve for $\bar{k}=20$ is also higher than that for $\bar{k}=10$, in contrast with what was observed for the average number of visited nodes (Figure 2(d)). Although visiting new nodes in scale-free networks is harder for those with higher average degree, it is easier to cover them because with high probability they are neighbors of the (more numerous) high degree nodes.

\subsection{Search Length}

This section shows results for the average search length $(\bar{h})$ achieved by SAW and RW in the three types of networks, with and without one-hop replication. Recall from the definition of the model (Section 2.4) that $\bar{h}$ is obtained from the average number of visited nodes $(V(h))$ for networks without one-hop replication, while it is obtained from the average number of covered nodes $(C(h))$ for networks with one-hop replication. We begin by comparing results for a single instance of the resource in the three types of networks. Then, we study the dependency of $\bar{h}$ on the number of instances of the resource in Section 3.5.

Figure 4 shows the average search length for SAW and RW in the three types of networks, with average degrees $\bar{k}=10,20,30$, with and without one-hop replication. Model predictions (bars) are 


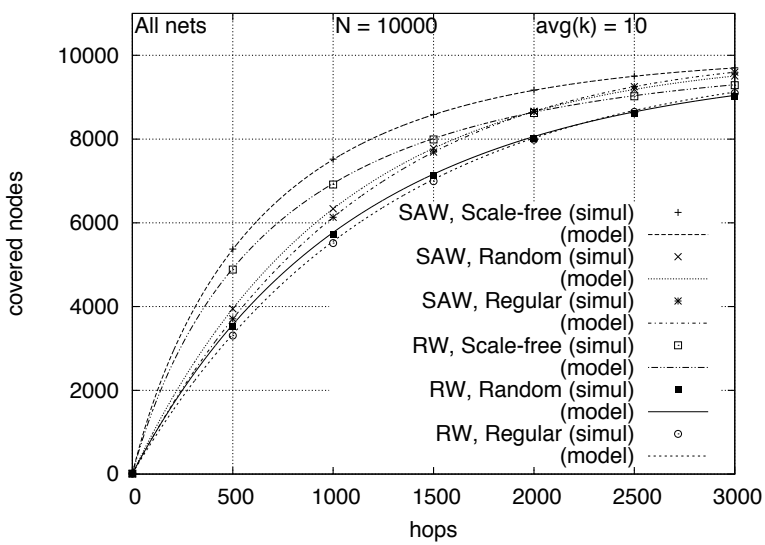

(a) All network types, $\bar{k}=10$

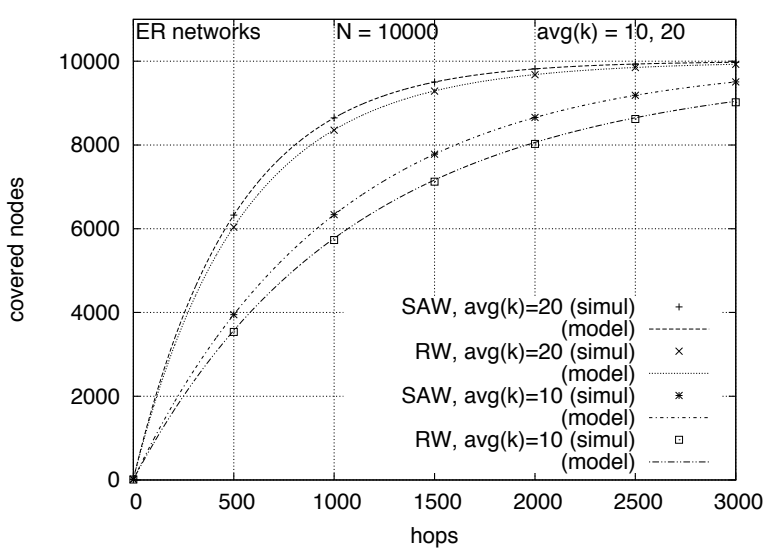

(c) ER random networks, $\bar{k}=10,20$

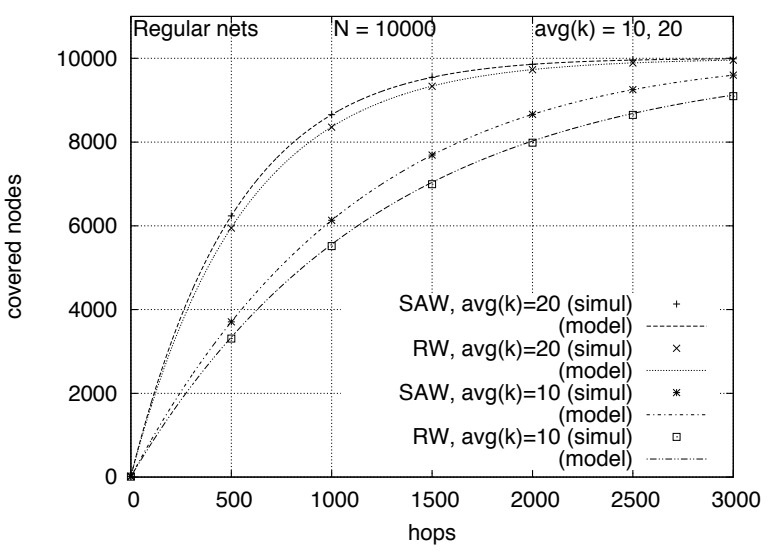

(b) Regular networks, $\bar{k}=10,20$

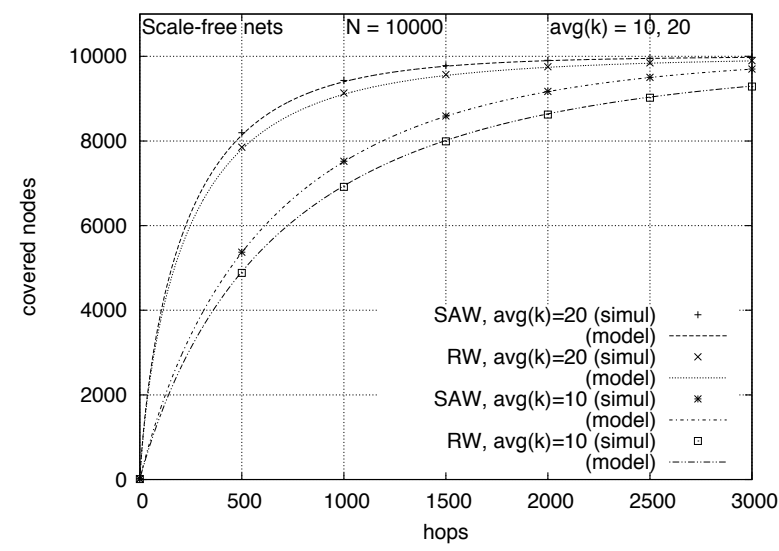

(d) Scale-free networks, $\bar{k}=10,20$

Figure 3: Average number of covered nodes of all degrees. 


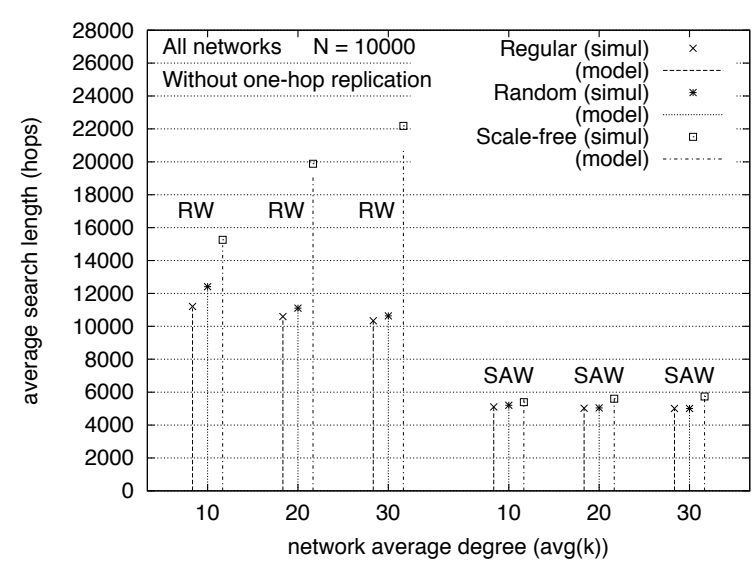

(a) Without one-hop replication.

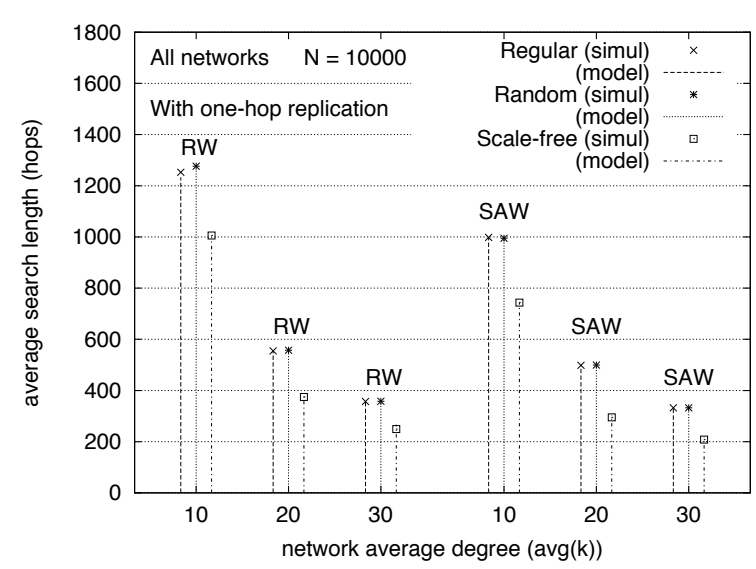

(b) With one-hop replication.

Figure 4: Average search length $(\bar{h})$ with a single resource instance.

in good agreement with simulation results (points). The SAW model registers errors with respect to the simulations smaller than $1.2 \%, 1.6 \%$ and $4.9 \%$ in regular, ER random and scale-free networks, respectively. More detail of these deviations are given in Table 3 in Section 3.4.4.

\subsubsection{Dependency on the one-hop replication feature}

If we first pay attention to the absolute values of $\bar{h}$ achieved by SAW in networks with $\bar{k}=10$, we notice that it is a little over half the network size in networks without one-hop replication, while it is around $10 \%$ the network size when the network has this feature. The former value agrees with Figure 2(a), where we observe that the SAW visits a new node at each hop most of the time, since the node that holds the instance of the resource sought has been randomly chosen. Likewise, the latter value agrees with Figure 3(a), where we observe that the number of nodes covered by the SAW at hop $h=1000$ is about half of the network size.

These graphs show two trivial results: $\bar{h}$ is smaller for SAW than for RW in the three types of networks; it is also smaller in networks with one-hop replication than in networks without this feature. It is more interesting to quantify the reduction in the average search length achieved by SAW with respect to RW for each network type. This information is presented in Table 2, where the reduction in $\bar{h}$ is given as $\left(\bar{h}_{R W}-\bar{h}_{S A W}\right) / \bar{h}_{R W} \cdot 100(\%)$. For networks without one-hop replication, the reduction is above $50 \%$, whereas for networks with this feature the reduction is smaller (above $20 \%$ ). This result is consistent with what we obtained in the two previous subsections for the 


\begin{tabular}{lcccc}
\cline { 2 - 5 } & & \multicolumn{3}{c}{ Reduction of $\bar{h}(\%)$} \\
\hline Network type & One-hop repl. & $\bar{k}=10$ & $\bar{k}=20$ & $\bar{k}=30$ \\
\hline \hline Regular & no & 54.46 & 52.57 & 51.57 \\
\cline { 2 - 5 } & yes & 20.32 & 10.10 & 6.95 \\
\hline \hline \multirow{2}{*}{ ER Random } & no & 57.92 & 54.60 & 52.89 \\
\cline { 2 - 5 } & yes & 22.47 & 10.40 & 7.17 \\
\hline \hline Scale-free & no & 64.67 & 71.82 & 74.16 \\
\cline { 2 - 5 } & yes & 26.07 & 21.31 & 19.88 \\
\hline
\end{tabular}

Table 2: Reduction of the average search length achieved by SAW with respect to that of RW.

average numbers of visited and covered nodes. There (see Figures 2(a) and 3(a)), we observed that there was a significant difference in curves of $V(h)$ for SAW and RW, while curves for $C(h)$ were almost coincident. This explains the smaller reductions of $\bar{h}$ in networks with one-hop replication.

\subsubsection{Dependency on the network type}

Going back to Figure 4, if we pay attention to the comparison among the three types of networks, we observe that both SAW and RW show different effects in networks with and without one-hop replication. In networks without one-hop replication, the algorithms achieve values of $\bar{h}$ in increasing order for regular, ER random and scale-free networks. The largest increment is registered for RW in scale-free networks. This is due to the existence of a large number of small degree nodes in scale-free networks. These nodes are more difficult to visit by the walks, especially by RW, yielding larger search lengths. This negative effect is almost totally compensated by SAW, since it tries to avoid already visited nodes, incrementing the probability of visiting low degree nodes. A consequence of this is the fact that SAW gets the largest reduction of $\bar{h}$ compared to RW in scale-free networks, as seen from Table 2 .

In networks with one-hop replication, values of $\bar{h}$ are similar for regular and ER random networks. Scale-free networks present smaller values, as opposed to what happened in networks without onehop replication. This is explained by the presence of very large degree nodes in scale-free networks. Although these nodes are few, they are visited with high probability, allowing many nodes to be covered without being visited, leading to reduced search lengths. This feature also yields larger reductions of $\bar{h}$ in scale-free networks, as verified from Table 2 . 


\subsubsection{Dependency on the average degree}

We focus again on Figure 4 to analyze the dependency of $\bar{h}$ on the average degree of the networks. For regular and ER random networks, a larger $\bar{k}$ yields a smaller $\bar{h}$, for both RW and SAW. For the former, the decrement is explained by the fact that the higher the degree of a node, the more probable it is to visit an unvisited neighbor of that node in the next hop. For the latter, the reduction comes from the fact that the probability that all the neighbors of a node have already been visited is lower if the degree of the node is higher. In networks without one-hop replication, the reduction in $\bar{h}$ when the average degree increases is small for RW and irrelevant for SAW. The reduction of $\bar{h}$ achieved by SAW with respect to RW slowly decreases with $\bar{k}$ (Table 2). In networks with one-hop replication, however, $\bar{h}$ is reduced by about one half when $\bar{k}$ is changed from 10 to 20, and about one third when $\bar{k}$ is changed from 20 to 30 , both for RW and SAW. The higher degree of the nodes allows walks to cover nodes faster, since nodes know about more neighbors. The reduction of $\bar{h}$ achieved by SAW with respect to RW decreases faster with $\bar{k}$ in this case than in networks without one-hop replication (Table 2).

The impact of the average degree in scale-free networks with one-hop replication is similar to that described for regular and ER random networks. However, the effect is reversed in networks without one-hop replication, where $\bar{h}$ increases for larger $\bar{k}$, both for RW and SAW. For RW, the average search length increases about $30 \%$ when $\bar{k}$ is changed from 10 to 20 , and about $10 \%$ when $\bar{k}$ is changed from 20 to 30 . The increment is less significant in SAW (under 5\%). This increment is again due to the larger number of high degree nodes (visited with high probability) in a network

with higher $\bar{k}$. This behavior makes SAW achieve a reduction of $\bar{k}$ with respect to RW that is growing with the average degree of the network, reaching $74 \%$ for $\bar{k}=30$ (Table 2 ).

\subsubsection{Dependency on the network size}

Finally, we analyze the dependency of the average search length on the size of the network. Simulation experiments for networks of $N=1000$ and $N=20000$ nodes have been added to the base experiments for $N=10000$ nodes presented so far. Figure 5 show the average search length obtained for regular, ER and scale-free networks as a function of their size. The average degree of all networks is $\bar{k}=10$. 


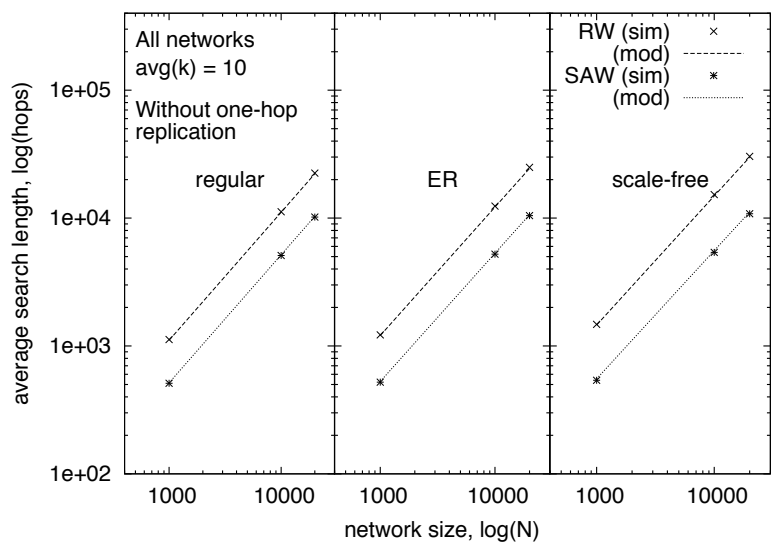

(a) Without one-hop replication.

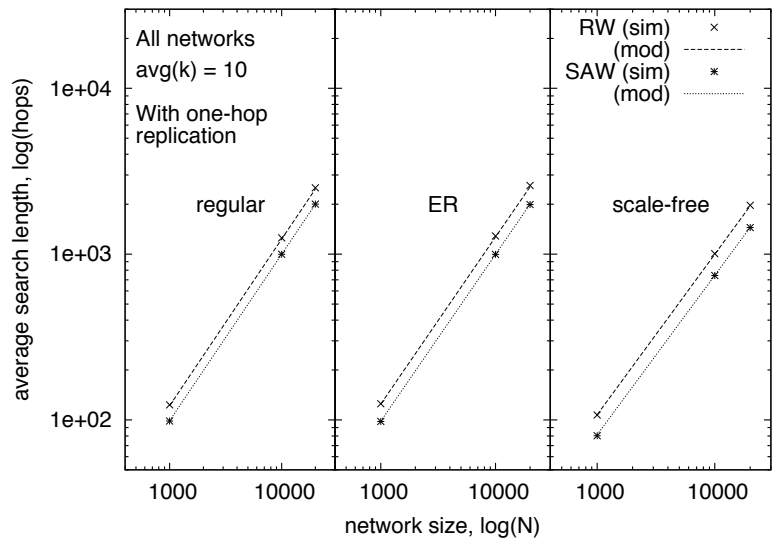

(b) With one-hop replication.

Figure 5: Average search length $(\bar{h})$ as a function of network size.

\begin{tabular}{lcccc} 
& & \multicolumn{3}{c}{$\bar{h}$ model prediction errors (\%) } \\
\hline Network type & One-hop repl. & $N=1000$ & $N=10000$ & $N=20000$ \\
\hline \hline \multirow{2}{*}{ Regular } & no & 0.88 & 1.17 & 1.35 \\
\cline { 2 - 5 } & yes & 0.38 & 0.09 & 0.15 \\
\hline \hline \multirow{2}{*}{ ER Random } & no & 1.29 & 1.57 & 1.51 \\
\cline { 2 - 5 } & yes & 0.53 & 0.06 & 0.44 \\
\hline \hline \multirow{2}{*}{ Scale-free } & no & 2.72 & 3.20 & 3.07 \\
\cline { 2 - 5 } & yes & 1.37 & 0.09 & 0.36 \\
\hline
\end{tabular}

Table 3: Relative errors of average search lengths predicted by the model with respect to simulation results, for networks with $\bar{k}=10$.

It is observed that the average search length is linear in the network size. As for the previous experiments, model predictions are in good agreement with simulation results. The magnitude of deviations depends on the network type, on the presence of the one-hop replication feature and on the network size. Table 3 presents these deviations relative to average search lengths registered in simulations.

\subsection{Several Instances of the Resource}

We now look at the dependency of the average search length on the number of instances of the resource sought present in the network $(R)$. Figures 6(a) to 6(c) show this dependency for the three types of networks, without one-hop replication, and for average degrees $\bar{k}=10,20$. Separately, to be able to adjust the scale of the $y$-axis adequately, Figures 6(d) to 6(f) show this dependency for 
networks with one-hop replication.

We observe that the reduction in $\bar{h}$ is large for the first additional resource instances, asymptotically tending to 0 as $R$ grows towards the network size. (In particular, we have checked that for SAW in networks without one-hop replication, this dependency is $\bar{h} \approx N /(R+1)$.) In networks without one-hop replication, the difference in the average search length achieved by SAW and RW quickly diminishes with the number of resource instances. A similar behavior can be observed in networks with one-hop replication and with average degrees 10 and 20.

In these experiments, SAW searches outperform RW searches in networks without one-hop replication regardless of their average degree. This is not true for networks with one-hop replication, where an increase in $\bar{k}$ has a large impact on the rate at which the network is covered. This results in a better performance of RW in networks with $\bar{k}=20$ than that of SAW in networks with $\bar{k}=10$.

\subsection{Variations of Network Averages}

As stated in Section 2, our model of the SAW is a mean-field analysis that produces a estimation of the average search length in networks with a given size and degree distribution. Of course, individual walks on a given network can yield large deviations from the predicted average value. To ensure the usefulness of the model when applied to an individual network (with the given degree distribution), the question that arises is now whether the choice of that particular topology can produce significant deviations. To answer this question, Figure 7 (for ER random networks) and Figure 8 (for scale-free networks) show deviations of network averages with respect to the value averaged over all networks (the average search length, $\bar{h}$ ). In particular, graphs show the standard deviation and the deviations of the maximum and minimum values of network averages with respect to $\bar{h}$. All deviations are given relative to $\bar{h}$. Recall from the description of the simulations (Section 3) that network averages are values averaged over the $10^{3}$ walks performed in each of the $10^{2}$ individual networks. From these results it can be stated that the average search length predicted by the model for a given size and degree distribution can be taken as a reasonably good approximation for any (randomly built) regular, ER or scale-free network with that size and degree distribution. 


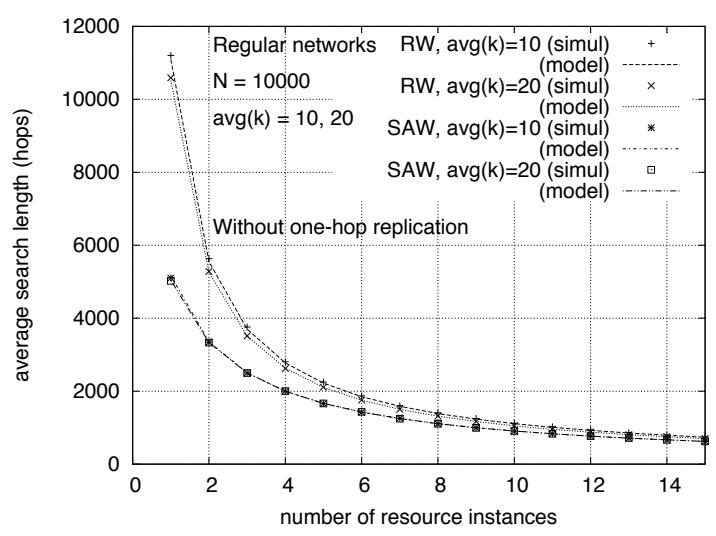

(a) Regular networks without one-hop replication

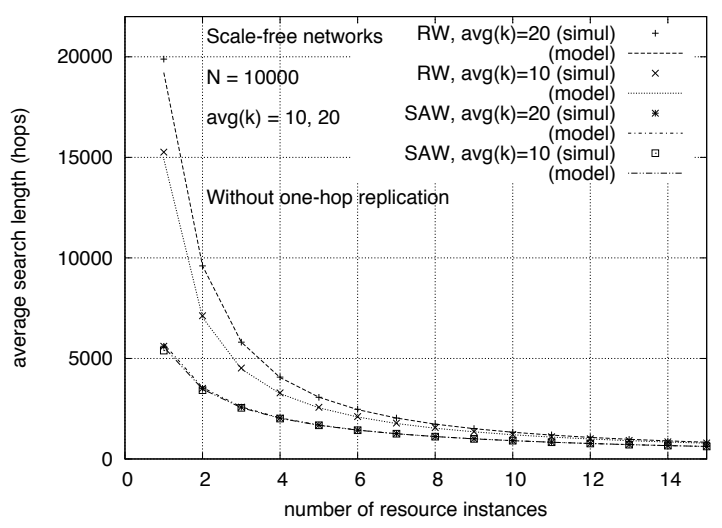

(c) Scale-free networks without one-hop replication

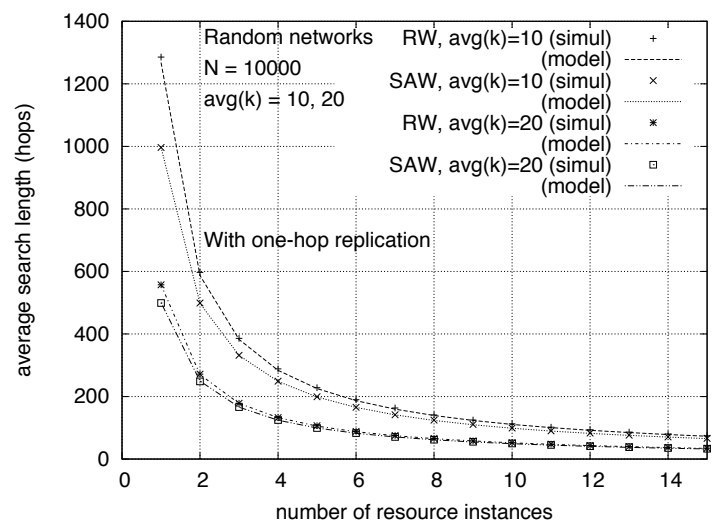

(e) ER random networks with one-hop replication

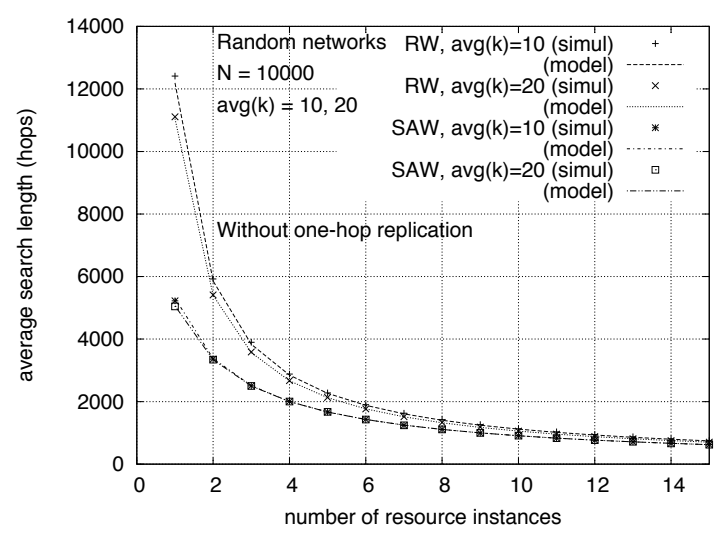

(b) ER random networks without one-hop replication

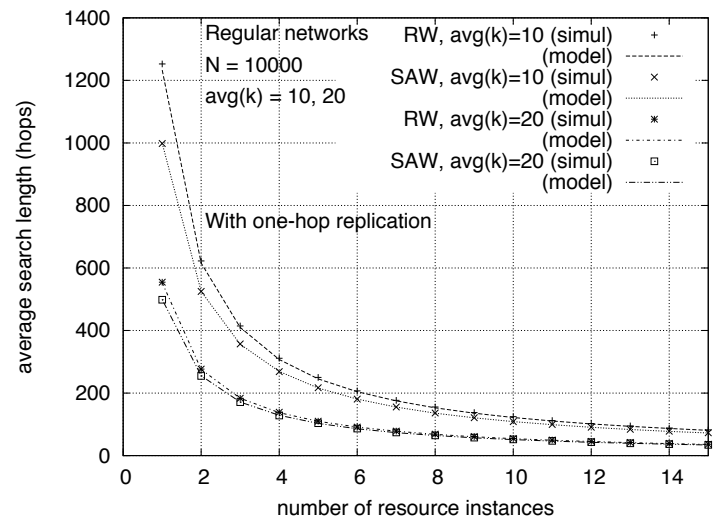

(d) Regular networks with one-hop replication

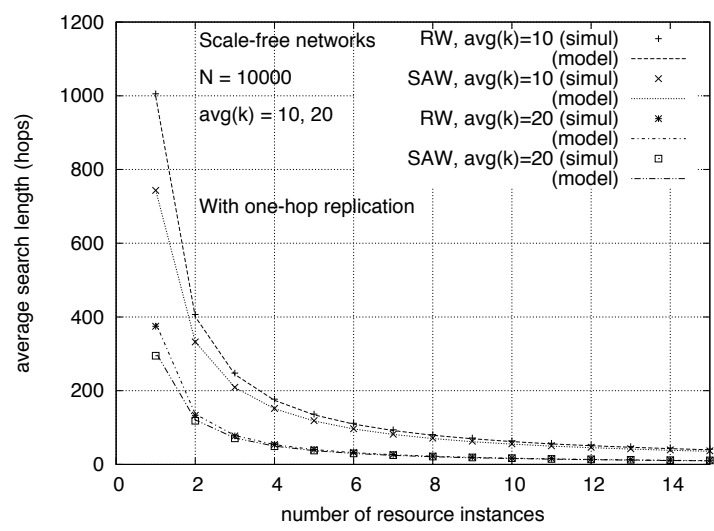

(f) Scale-free networks with one-hop replication

Figure 6: Average search length for several resource instances in networks with $\bar{k}=10,20$. 


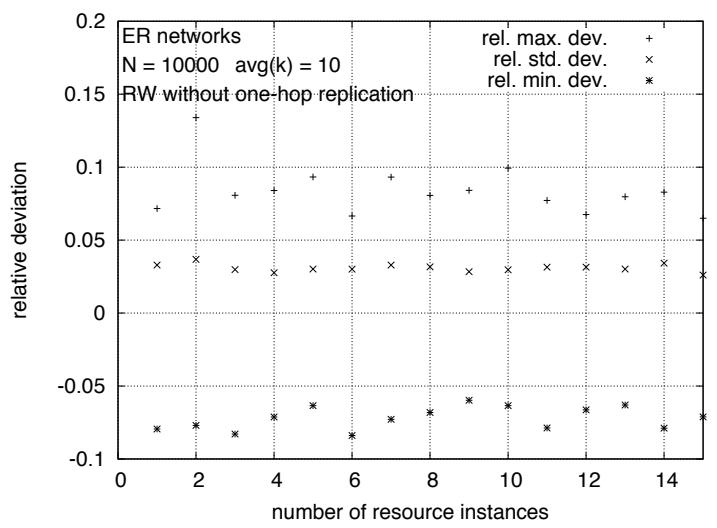

(a) RW without one-hop replication

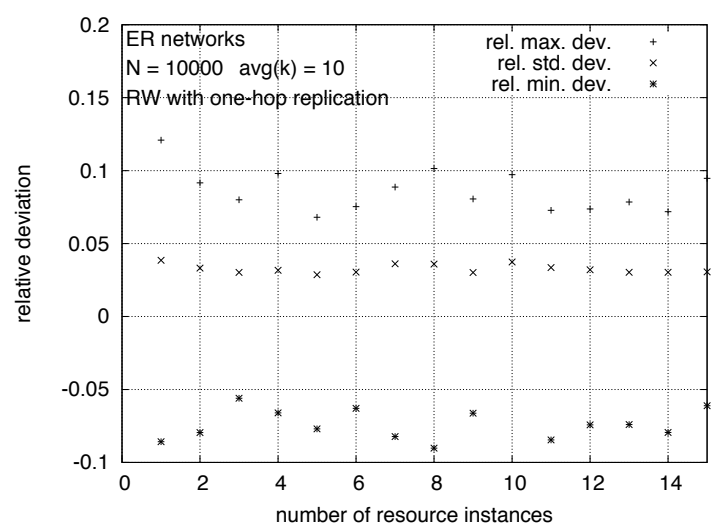

(c) RW with one-hop replication

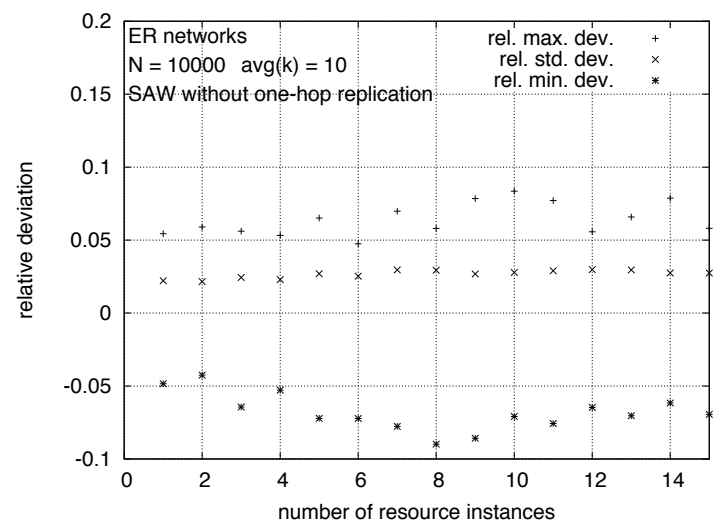

(b) SAW without one-hop replication

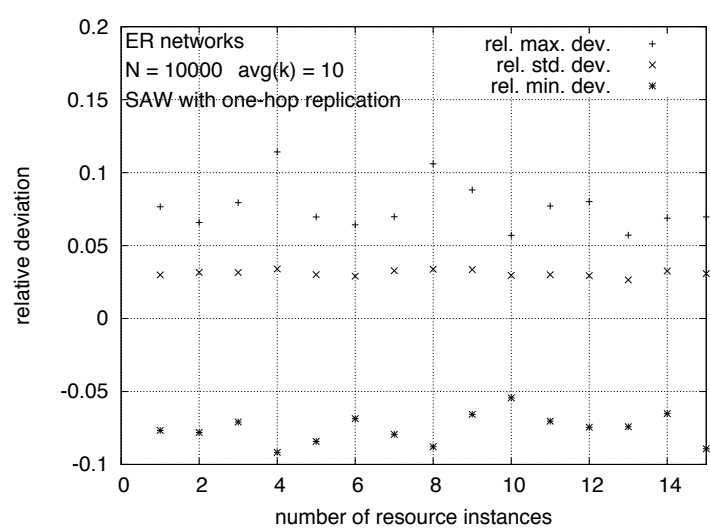

(d) SAW with one-hop replication

Figure 7: Relative deviations of network averages for search lengths in ER random networks with $\bar{k}=10$. 


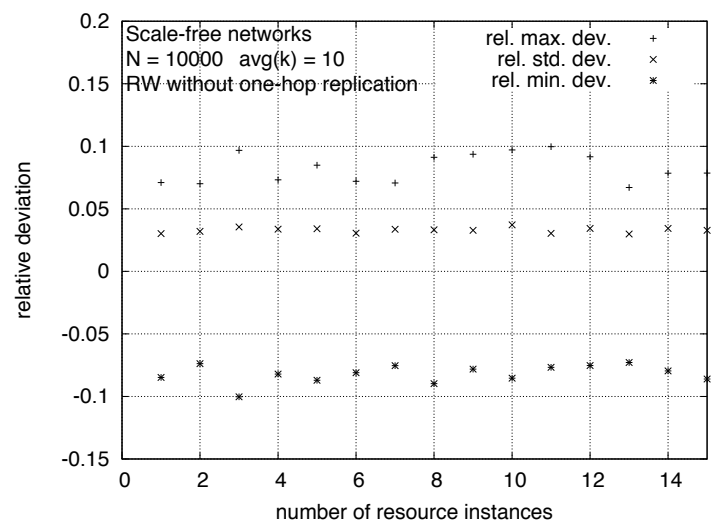

(a) RW without one-hop replication

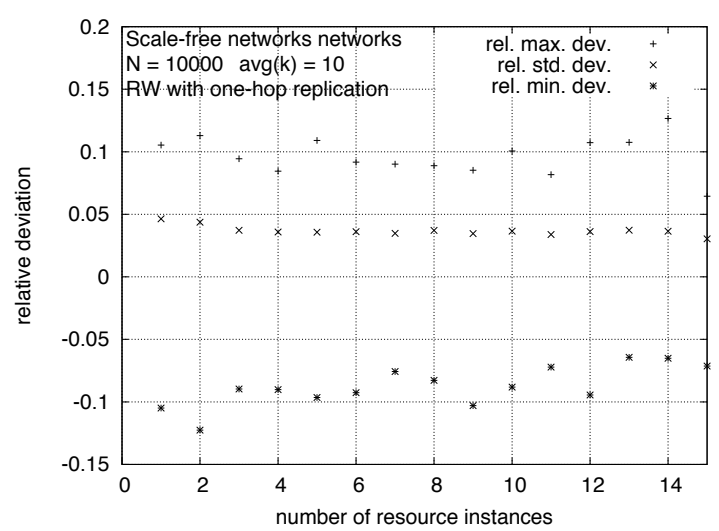

(c) RW with one-hop replication

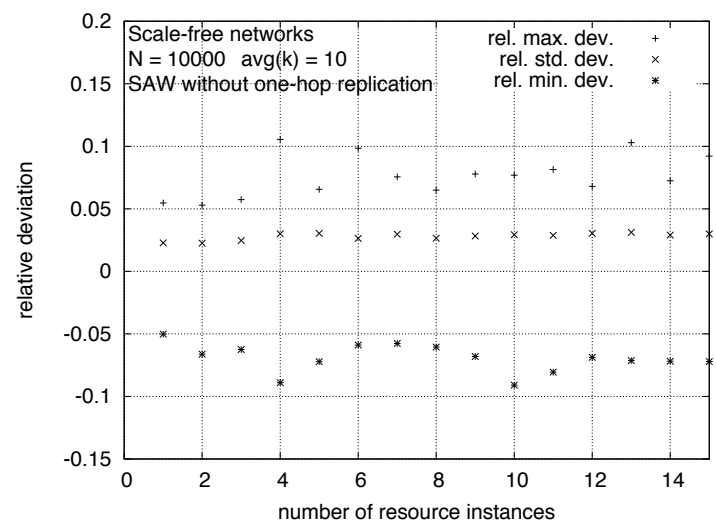

(b) SAW without one-hop replication

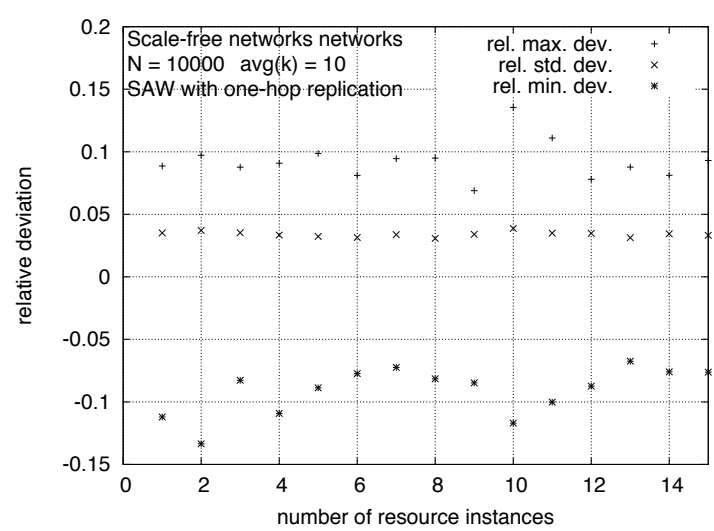

(d) SAW with one-hop replication

Figure 8: Relative deviations of network averages for search lengths in scale-free networks with $\bar{k}=10$. 


\section{Conclusions}

We have proposed a mean-field model to estimate the average search length in randomly built networks with a given size and degree distribution. The model considers the possible use of one-hop replication and the existence of multiple resource instances. We have empirically evaluated the model by generating networks of three types (regular, ER random and scale-free) and simulating searches in them. We have found that the estimates are very accurate. When using SAW in networks with one-hop replication, the average search length decreases with the increase in average degree for the three types of networks. In networks without one-hop replication, however, the impact of average degree on the average search length is small, with slight decrements for ER random and regular networks and slight increments for scale-free networks. Regarding the behavior with respect to multiplicity of resource instances, the average search length is roughly inversely proportional to the number of instances.

We have also simulated RW, with and without one-hop replication and with multiple resource instances. The conclusion is that SAW has a much smaller average search length when one-hop replication is not available, especially in scale-free networks, where the reduction increases as the average degree of the network grows. In networks with one-hop replication, reductions of average search length are only significant for low average degrees, decreasing as the average degree grows. The behavior with respect to the number of resource instances is similar to that of SAW.

An interesting future line to continue this work is to evaluate the search performance of variations of the SAW, using different probability distributions for choosing the neighbor to visit next, e.g., based on combinations of its degree and of the number of times it has previously been visited or covered.

\section{References}

\section{References}

[1] L.A. Adamic and B.A. Huberman, Zipf's law and the internet, Glottometrics 3 (2002), 143-150.

[2] L.A. Adamic, R.M. Lukose, A.R. Puniyani, and B.A. Huberman, Search in power-law networks, Physical Review E 64 (2001). 
[3] M. Alanyali, V. Saligrama, and O. Savas, A random-walk model for distributed computation in energy-limited networks, Proc First Workshop Informat Theory its Application, 2006, pp. .

[4] D.J. Aldous, Lower bounds for covering times for reversible markov chains and random walks, J Theoret Probability 2 (1989), 91-100.

[5] D. Amit, G. Parisi, and L. Peliti, Asymptotic behavior of the 'true' self-avoiding walk, Physical Review B 27 (1983), 1635-1645.

[6] A.L. Barabási and E. Bonabeau, Scale-free networks, Sci American 288 (May 2003), 60-69.

[7] G. Barnes and U. Feige, Short random walks on graphs, Proc Twenty-fifth Ann ACM Symp Theory Comput (STOC 93), ACM Press, 1993, pp. 728-737.

[8] N. Bisnik and A. Abouzeid, Modeling and analysis of random walk search algorithms, Proc Second Int Workshop Hot Topics in Peer-to-Peer Syst (Hot-P2P 2005), New York, New York, United States, IEEE Computer Society, 2005, pp. 95-103.

[9] G. Brightwell and P. Winkler, Maximum hitting time for random walks on graphs, Random Structures Algorithms 1 (1989), 263-276.

[10] J. Candia, P.E. Parris, and V.M. Kenkre, Transport properties of random walks on scalefree/regular lattice hybrid networks, J Stat Phys 129 (2007), 323-333.

[11] Y. Chawathe, S. Ratnasamy, N. Lanham, and S. Shenker, Making gnutella-like p2p systems scalable, Proc 2003 Conference Appl, Technologies, Architectures, Protocols Comput Commun (SIGCOMM), Karlsruhe, Germany, 2003, pp. 407-418.

[12] V. Cholvi, P.A. Felber, and E.W. Biersack, Efficient search in unstructured peer-to-peer networks, Proc Sixteenth Ann ACM Symp Parallelism in Algorithms Architectures, Barcelona, Spain, 2004, pp. 271-272.

[13] L. da Fontoura Costa and G. Travieso, Exploring complex networks through random walks, Physical Review E 75 (2007).

[14] S. Dolev, E. Schiller, and J.L. Welch, Random walk for self-stabilizing group communication in ad hoc networks, IEEE Trans Mobile Comput 5 (2006), 893-905. 
[15] U. Feige, A tight lower bound on the cover time for random walks on graphs, Random Structures Algorithms 6 (1995), 433-438.

[16] S. Fortunato and A. Flammini, Random walks on directed networks: The case of pagerank, e-print physics (2006).

[17] C. Gkantsidis, M. Mihail, and A. Saberi, Random-walks in peer-to-peer networks: Algorithms and evaluation, Performance Evaluation 63 (2006), 241-263.

[18] C.P. Herrero, Self-avoiding walks on scale-free networks, Physical Review E 71 (2005).

[19] B.D. Hughes, Random walks and random environments Vol. 1, Clarendon Press, Oxford, 1995.

[20] J.D. Kahn, N. Linial, N. Nisan, and M.E. Saks, On the cover time of random walks on graphs, J Theoret Probability 2 (1989), 121-128.

[21] T. Kuczek and K. Crank, On a self-avoiding random walk, Indian J Stat 56 A (1994), 54-66.

[22] C. Law and K.Y. Siu, Distributed construction of random expander networks, Proc Twentysecond Ann Joint Conference IEEE Comput Commun Societies (INFOCOM 2003), Vol. 3, 2003, pp. 2133-2143.

[23] S.H. Lee, P.J. Kim, and H. Jeong, Statistical properties of sampled networks, Physical Review E $73(2006)$.

[24] L. Lovász, "Random walks on graphs: A survey," Combinatorics, Paul Erdős is eighty, Bolyai Society Mathematical Studies, 2, Keszthely (Hungary), 1993, Vol. 2, pp. 1-46.

[25] Q. Lv, P. Cao, E. Cohen, K. Li, and S. Shenker, Search and replication in unstructured peer-topeer networks, Proc Sixteenth Int Conference Supercomputing, New York, New York, United States, 2005, pp. 84-95.

[26] Q. Lv, S. Ratnasamy, and S. Shenker, Can heterogeneity make gnutella scalable?, Revised papers from First Int Workshop Peer-to-Peer Syst, Cambridge, United States, 2002, pp. 94103. 
[27] I. Mabrouki, X. Lagrange, and G. Froc, Random walk based routing protocol for wireless sensor networks, Proc Second Int Conference Performance Evaluation Methodologies Tooks (ValueTools '07), ICST (Institute for Computer Sciences, Social-Informatics and Telecommunications Engineering), 2007, pp. 1-10.

[28] N. Madras and G. Slade, The self-avoiding walk, Birkhäuser, Boston, 1996.

[29] G.S. Manku, M. Naor, and U. Wieder, Know thy neighbor's neighbor: The power of lookahead in randomized p2p networks, Proc 36th Ann ACM Symp Theory Comput (STOC 2004), ACM Press, 2004, pp. 54-63.

[30] R. Motwani and P. Raghavan, Markov chains and random walks chapter 6, pp. 127-160, Cambridge University Press 1995.

[31] M.E.J. Newman, A.L. Barabási, and D.J. Watts, The structure and dynamics of networks, Princeton University Press, 2006.

[32] M.E.J. Newman, S.H. Strogatz, and D.J. Watts, Random graphs with arbitrary degree distributions and their applications, Physical Review E 64 (2001).

[33] S.A. Pandit and R.E. Amritkar, Random spread on the family of small-world networks, Physical Review E 63 (2001).

[34] M. Ripeanu, Peer-to-peer architecture case study: Gnutella network, Proc First Int Conference Peer-to-Peer Comput, 2001, pp. 99-100.

[35] L. Rodero-Merino, A. Fernández Anta, L. López, and V. Cholvi, Performance of random walks in one-hop replication networks, Comput Networks 54 (2010), 781-796.

[36] N. Sadagopan, B. Krishnamachari, and A. Helmy, Active query forwarding in sensor networks, Ad hoc Networks 3 (2005), 91-113.

[37] N. Sarshar and V. Roychowdhury, Scale-free and stable structures in complex ad-hoc networks, Physical Review E 69 (2004). 
[38] G. Slade, The self-avoiding walk: A brief survey, Revised May 28, 2010. To appear in Surveys in Stochastic Processes, Proceedings of the 33rd SPA Conference in Berlin, 2009, to be published in the EMS Series of Congress Reports, eds. J. Blath, P. Imkeller, S. Roelly.

[39] G. Slade, The diffusion of self-avoiding random walk in high dimensions, Commun in Math Phys 110 (1987), 661-683.

[40] B. Tadić, Adaptive random walks on the class of web graphs, Eur Physical J B 23 (2001), $221-228$.

[41] S.J. Yang, Exploring complex networks by walking on them, Physical Review E 71 (2005).

[42] D. Zuckerman, A technique for lower bounding the cover time, Proc Twenty-second Ann ACM Symp Theory Comput, 1990, pp. 254-259. 